This report was prepared as an account of work sponsored by an ageacy of the United States Gowernment. Neither the United States Government nor any asency thereof, nor any of their employees, makes any warranty, express or implied, or asumes any leanl liability or responsbility for the sccuracy, completeneas, or usefulness of any information, apparatus, product, or process disclosed, or represents that its use would not infringe privately owned rights. Reference herein to any specific commercial product, process, or service by trade name, trademark, manufacturer, or otberwise does not necessarily constitute or imply its eadorsement, recommendation, or favoring by the United States Government or any agency thereof. The views and opinions of authors expressed herein do not necessarily state of reflect those of the United States Gowernment or any agency thereor.

\title{
ANALYSIS OF NEUTRON DATA IN TIE RESONANCE REGION VIA THE COMPUTER CODE SAMMY*
}

\author{
N. M. LARSON \\ Oak Ridge National Laboratory, Oak Ridge, Tenn. 37831 USA
}

\begin{abstract}
Procedures for analysis of resonance neutron cross-section data have bcen implemented in a state-of-the-art computer code SAMMY, ${ }^{1-3}$ developed at the Oak Ridge Electron Linear Accelerator (ORELA) at Oak Ridge National Laboratory. A unique feature of SAMMY is the use of Bayes' equations to determine "best" values of parameters, which permits sequential analysis of data sets (or subsets) while giving the same results as would bo given by a similtaneous analysis. Another important feature is the inclusion of data-reduction parameters in the fitting procedure. Other features of SAMMY are also described.
\end{abstract}

\section{INTRODUCTION}

In 1979 the need for a multilevel R-matrix code incorporating both (1) the best features of the code MULTI ${ }^{4}$ and (2) the use of Bayes' method was recognized at ORELA. The resulting code, SAMMY, ${ }^{1-3}$ has evolved continuously over the intervening years in an effort to meet the requirements for analysis of ORELA (and other) data. Four SAMMY analyses are reported at this conference; ; $^{-8}$ a list of publications of other SAMMY analyses is available from the author. In this paper we concentrate on describing options available in SAMMY, rather than on discussing results of specific analyses.

\section{BAYES' METHOD}

The major distinguishing feature of SAMMY is its use of Bayes' method rather than least squares to update parameter values and to determine the covariance matrix for those parameters. Bayes method may be viewed as a generalized leastsquares technique. for which prior information is incorporated into tlac equations.

\footnotetext{
- Kesearcth sponsored hy the U.S. Bepartment of tenergy under contract DI:-ACOS-K4OR 21400) with Marrtun Marrietta linergy Systems, Inc.
} 


\section{N. M. L.ARSON}

(Equations and deviations thereof are presented in the SAMMY users' manuals. ${ }^{1-3}$ ) As a result, SAMMY has several advantages over codes using least-squares techniques: (1) Inclusion of data covariances is straightforward. (2) If a varied parameter is irrelevant to the data being analyzed, both its value and its uncertainty will be unchanged by the analysis. With least-squares techniques, the value of such a parameter is of ten subject to unreasonable changes. (3) Results from one analysis may be communicated to a subsequent analysis by using the output parameter values and covariance matrix from the first analysis as input to the second. Output parameter values from the second analysis then produce a valid fit for both data sets. (4) As a consequence of item (3). a series of sequential analyses of data subsets may be used to obtain results effectively equivalent to those obtained from a single simulianeous analysis of the combined data sets. The overall fit for analysis of a data set will lie within experimental uncertainties whether the data set was analyzed in one piece or in several smaller pieces.

\section{DATA-REDUCTION PARAMETERS}

A recent innovation in SAMMY is the ability to include data-reduction parameters as well as theory parameters in the fitting procedure. Seven parameters (sample thickness, normalization, and five parameters for energy-dependent backgrounds) are treated explicitly, in the sense that users need merely specify values and uncertainties and set flags to indicate which parameters are to be varied.

In addition to treating some parameters explicitly, SAMMY has available a general scheme to vary any data-reduction parameter. Partial derivatives of the reduced data with respect to the data-reduction parameters are fed into SAMMY alung with the data and the statistical uncertainties. (For a method whereby these partial derivatives may be generated, see ref. 9.) SAMMY's fitting procedure (Bayes' method) then updates values and uncertainties for both kinds of parameters. This ability to include data-reduction parameters as part of the fitting procedure allows the correct treatment of data uncertainties, even for very large data sets. The alternative is to treat the data covariance matrix in its fu!l form (off-diagonal). which requires that all correlated data be analyzed simultaneously. This is inpractical for very large data sets such as those generated at ORELA.

\section{OTHER FEATURES OF SAMMY}

The Reich-Moorc approximation to muleilevel R-matrix theory is used to generate theoretical cross sections in SAMMY: alternatively. multilevel Breit-Wigner may be used. Contributions from resonances outside the energy region being analyzed may be parameterized cither as "dummy" resonances or via a logarithmic function. Virtually any parameter of the $R$-matrix or external $R$-function may be varied. 


\section{COMPUTER CODE SAMMY}

Broadening of both cross sections and partial derivatives is accomplished by numerically folding a Doppler- and/or resolution-broadening function with the theoretical cross section or derivative. The Doppler broadening function is a

Gaussian of width proportional to $\sqrt{E T_{\text {eff. }}}$. Resolution broadening appropriate for a white source is included as a Gaussian function, an exponential function, or a convolution of the two. A more general parameterization of resolution broadening will scon be incorporated. allowing the user to more accurately mimic actual experimental conditions. Broadening parameters may be varied as part of the fitting procedure.

Data types which may be analyzed with SAMMY include transmission, total cross section, elastic and/or inclastic scattering. fission, capture, or absorption cross sections. (Multiple-scattering corrections for capture are not yet incorporated into SAMMY but are planned for the near future.) SAMMY permits analysis of several types of data simultaneously, though such analyses are needed only when data sets are correlated.

Input to SAMMY, though necessarily somewhat complex, was designed to be user-friendly. Where possible, descriptive alphanumeric phrases are used rather than numerical flags whose meanings are more easily misinterpreted. SAMMY tests all input for internal inconsistencies and other obvious etrors; in most cases SAMMY will inform the user what crror needs correcting.

SAMMY is written in FORTRAN, with few machine-dependent constructions. Dynamic allocation of array storage and the use of temporary files permit SAMMY to run large jobs on relatively small computers. Versions of SAMMY have been adapted for DEC PDP-10, VAX, and IBM computers. Thorough documentation is available, both via extensive comment cards in the FORTRAN and via the users' $g_{1} \cdot ?, 1 \cdot 3$

\section{REFERENCES}

1. N. M. Larson and F. G. Perey, ORNL/TM-7485 (1980).

2. N. M. Larson, ORNL,TM-9179 (1984).

3. N. M. Larson, ORNL/TM-9179/R1 (1985).

4. G. F. Auchampaugh, LA-5473-MS (1974).

5. F. G. Percy. Status of the Parameters of the 1.15-keV Resonance of Fe-56. (IA05).

6. M. M. Salah, i. A. Harvey. N. W. Ilill, A. Z. Ilussein and I:. G. Percy.

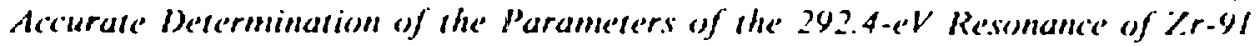
and the $301 / .3-$ - V Resomance of $Z r-90,(J \wedge 22)$.

7. Ci. de Saussure, R. B. Peres, and R. L. Macklin, R-mastrix Analysis of the Pu2.39 Neuron Crosss Secrion. $(J C 39)$. 
N. M. LARSON

8. C. M. Perey, F. G. Percy, J. A. Ilarvey. N. W. Hill, and R. L. Macklin, Ni-58 $+n$ Transmission. (apture and Differential Elastic Scattering Data Analysis in the Resonance Region, ( JC40).

9. N. M. Larson, Uncertainy Propagation from Raw Data to Final Results. (JC28). 